\title{
Ginsenoside Rg1 protects starving H9c2 cells by dissociation of Bcl-2-Beclin1 complex
}

\author{
Dan $\mathrm{Li}^{1+}$, Jun Wang ${ }^{2 \dagger}$, Jincai Hou', Jianhua Fu', Dennis Chang ${ }^{3}$, Alan Bensoussan ${ }^{3}$ and Jianxun Liư ${ }^{1 *}$
}

\begin{abstract}
Background: Autophagy can result in cellular adaptation, as well as cell survival or cell death. We investigated how ginsenoside $\operatorname{Rg} 1$ (G-Rg1) regulates the relationship between autophagy and apoptosis induced by continuous starvation.

Methods: $\mathrm{H} 9 \mathrm{C} 2$ cells under continuous starvation were treated with or without ginsenoside $\mathrm{Rg} 1$, and autophagy and apoptosis related proteins were assessed over a continuous time course by Western blot. Dynamic fluorescence intensity of green fluorescent protein (GFP)-LC3 was used to assess autophagosome formation by live cell imaging. Cyan fluorescent protein (CFP) -Beclin1(BECN1) and yellow fluorescent protein (YFP) -BCl-2 were co-transfected into cells to observe ginsenoside $\mathrm{Rg} 1$ regulation of $\mathrm{BECN1/BCl-2}$ interaction using Fluorescence Resonance Energy Transfer (FRET). Immunoprecipitation was also used to assess BECN1/BCl-2 interaction over a continuous time course.

Results: In H9c2 cells, starvation induced both apoptosis and autophagy. Cell apoptosis was significantly attenuated in ginsenoside Rg1-treated conditions, while autophagy was promoted. Ginsenoside Rg1 weakened the interaction between Beclin1 and $\mathrm{BCl}-2$, inhibiting apoptosis while promoting autophagy. Our results suggest that autophagy is beneficial to starved cardiac cells over a period of time. Furthermore, we describe the effect of ginsenoside Rg 1 on the relationship between autophagy and apoptosis during starvation.
\end{abstract}

Conclusions: Our findings provide valuable evidence for employing ginsenoside Rg1 as a specific promoter of autophagy and inhibitor of apoptosis.

Keywords: Ginsenoside Rg1, Autophagy, Apoptosis, Dynamic relationships, Cardiomyocytes

\section{Background}

Although the concept of autophagy was established decades ago, the exact role it plays in pathological processes remains unclear. In cardiomyocytes, autophagy plays a dual role, demonstrating either protective or harmful effects under different pathological conditions [1-5]. Some studies suggest that autophagy protects cells from oxidative stress and apoptosis, a key survival mechanism, and maintains energy homeostasis and viability in cell starvation models [6-11]. However, other studies suggest that autophagy can enhance cardiomyocyte damage induced

\footnotetext{
*Correspondence: ljxunljxun@163.com; liujx0324@sina.com

${ }^{\dagger}$ Equal contributors

${ }^{1}$ Institute of Basic Medical Sciences, Xiyuan Hospital, China Academy of Chinese Medical Sciences, Beijing Key Laboratory of Pharmacology of

Chinese Materia Medica, Hai Dian District, Beijing, China

Full list of author information is available at the end of the article
}

by high glucose levels [12] and ischemia/reperfusion [13]. It is somewhat surprising that even under the same pathological condition (e.g. starvation), autophagy can exert both pro-survival and pro-death effects, two seemingly opposing roles. It has been demonstrated that excessive autophagy leads to cell death under starvation conditions. Thus, the extent of autophagy appears to be critical for determining whether it will play a protective or harmful role [14]. However, little research has been done to assess the dynamic process of autophagy during starvation. It is currently unclear if the level of autophagy changes at different time points during starvation and, if so, how this might impact cardiomyocyte health. Therefore, pharmacological effects should be evaluated in a dynamic fashion, rather than relying on a single time point. 
Ginsenosides are a group of nontoxic, bioactive components derived from the rhizome of Panax ginseng, one of the mostly commonly used Chinese herbs. Growing evidence suggests that ginsenosides possess protective abilities against coronary artery disease, myocardial ischemia, cardiac hypertrophy, heart failure and arrhythmia [15-20]. Ginsenoside Rg1, one of the main members of the ginsenoside family, has been shown to promote angiogenesis [21-24], to exert anti-hypertension effects [25] and to positively affect myocardial remodeling [26-28]. A recent study indicated that $\operatorname{Rg} 1$ inhibits apoptosis by regulating autophagy [13], but the exact mechanism remains unclear. Furthermore, no study to date has assessed the dynamic regulation of autophagy by $\operatorname{Rg} 1$.

Autophagy and apoptosis can occur simultaneously in response to starvation, and the interplay between autophagic and apoptotic pathways is emerging as a crucial process in determining the initiation of programmed cell death $[29,30]$. Beclin 1 , a Bcl-2 interacting protein, has been implicated as an important regulator of autophagy [31]. Indeed, the suppression of Beclin 1 expression impairs autophagy and sensitizes the cells to starvation-induced apoptosis [32]. Beclin 1 interacts with anti-apoptotic multi-domain proteins of the Bcl-2 family, in particular Bcl-2 and its homologue $\mathrm{Bcl}-\mathrm{X}(\mathrm{L})$, by virtue of its $\mathrm{BH} 3$ domain, an amphipathic alpha-helix that binds to the hydrophobic cleft of $\mathrm{Bcl}-2 / \mathrm{Bcl}-\mathrm{X}(\mathrm{L})$ $[33,34]$. It has been shown that the interaction between Bcl-2 and Beclin1 leads to the inhibition of autophagy by interfering with the formation and activity of the autophagy promoter complex, Beclin $1 / \mathrm{hVps} 34$ [35]. It has not yet been determined whether ginsenoside Rg1 regulates autophagy through Bcl-2 and Beclin1.

In the present study, we sought to address the dynamic regulation of ginsenoside $\operatorname{Rg} 1$ on autophagy for protecting cardiomyocytes from apoptosis under prolonged starvation and the related molecular mechanism by which ginsenoside $\mathrm{Rg} 1$ regulates autophagic activity.

\section{Methods}

\section{Materials}

Commercial antibodies and chemicals were purchased from the following sources: anti-LC3 (L7543) and anticaspase 3 (C8487) antibodies, rapamycin (Rap;R8781) and 3-methyladenine (3-MA;M9281) were purchased from Sigma-Aldrich; anti-Beclin1 (3738s), anti-Bcl-2 (2870s) and anti-GAPDH (5174s) antibodies were purchased from Cell Signaling Technology. Ginsenoside Rg1 (Purity > 95 \%, 110703-201027) were chemically standardized products obtained from the National Institutes for Food and Drug Control, which was validated by fingerprint chromatographic methodologies.

\section{Starvation model in $\mathrm{H} 9 \mathrm{c} 2$ cells and drug treatment}

The rat ventricular cell line $\mathrm{H} 9 \mathrm{c} 2$ was purchased from the cell bank of the Chinese Academy of Medical Sciences (Beijing, China). Cells were cultured in Dulbecco's modified Eagles medium (DMEM, Hyclone) containing $10 \%$ fetal bovine serum (GIBCO), $40 \mathrm{U} / \mathrm{ml}$ penicillin and 40 $\mathrm{U} / \mathrm{ml}$ streptomycin in a humidified atmosphere of $95 \%$ air $/ 5 \% \mathrm{CO}_{2}$ at $37{ }^{\circ} \mathrm{C}$. Cells were grown to $80 \%$ confluency before being exposed to starvation. To create starvation conditions, cardiac myocytes were washed three times with phosphate-buffered saline (PBS) and were incubated in glucose-free, serum-free DMEM (GIBCO, 11966) at $37^{\circ} \mathrm{C}$ for $0,30,60,90,120,150,180,210$ or 240 min or until cell death. Cells cultured in nutrient-rich medium were used as a control. For the active treatment study, cells were pre-treated with ginsenoside $\operatorname{Rg} 1$ (Rg1) for $12 \mathrm{~h}$ in nutrient-rich medium and then were treated with ginsenoside $\operatorname{Rg} 1$ for $0,30,60,90,120,150,180,210$ or $240 \mathrm{~min}$ or until cell death in glucose-free, serum-free DMEM. Rg1 was first dissolved in DMSO and then diluted to the final concentration in culture medium (final DMSO concentration $<1 \%$ ).

\section{Cell viability assay}

Cells were seeded in 96-well plates $\left(2 \times 10^{4}\right.$ cells per well). After $48 \mathrm{~h}$, cell viability was measured using the 3-(4,5)dimethylthiahiazo (-z-y1)-3,5-diphenytetrazoliumromide (MTT) assay at different time points (30, 60, 90, 120, 150, 180,210 or $240 \mathrm{~min}$ ) following glucose-deprivation. MTT was dissolved and sterilized in PBS at $0.5 \mathrm{mg} / \mathrm{ml}$, and 100 $\mu \mathrm{L}$ of this solution was added to each well. The plate was incubated at $37^{\circ} \mathrm{C}$ for $4 \mathrm{~h}$ before the media was removed. Approximately $200 \mu \mathrm{L}$ of dimethyl sulfoxide (DMSO) was added into each well, and the plate was gently rocked for 10 min to dissolve the dark blue MTT crystals. Cell viability was expressed as optical density (OD), which was detected by use of a microplate reader at $490 \mathrm{~nm}$. The experimental groups included one normal control group (starvation for 0 min), eight starvation model groups (starvation for 30, 60, $90,120,150,180,210$ or $240 \mathrm{~min}$ ) and twenty-seven ginsenoside Rg1 treatment groups (treated with 25, 50 or 100 $\mu \mathrm{M}$ ginsenoside Rg1 for each time point).

\section{Live cell imaging system for green fluorescent protein (GFP)-LC3}

Live cell imaging experiments were carried out for one model group and one drug treatment group. The treatment group was treated with $100 \mu \mathrm{M}$ ginsenoside Rg1, as described above. The $\mathrm{H} 9 \mathrm{c} 2$ cells were transfected with GFP-LC3 expression plasmids using an electroporation apparatus (BTX). The electroporation parameters used were as follows: square-wave, $100 \mathrm{~V} / \mathrm{cm}, 0.5 \mathrm{~ms}$ and 5 impulses. Electroporation was carried out in low conductivity electroporation buffer $\left(10 \mathrm{mM}\right.$ phosphate, $1 \mathrm{mM} \mathrm{MgCl}_{2}, 250$ 
$\mathrm{mM}$ sucrose, $\mathrm{pH}$ 7.4). Cells were allowed to recover for 20 min following electroporation and before being placed in normal medium and returned to normal culture conditions. After $48 \mathrm{~h}$, the transfected cells were glucose deprived for $24 \mathrm{~h}$. The dynamic changes in color and fluorescence intensity were evaluated in the same cell selected at different times using the live cell imaging system (Olympus).

\section{Terminal dexynucleotidyl transferase-mediated dUTP nick} end labeling (TUNEL) assay

TUNEL experiments were carried out for nine model and nine treatment groups. The treatment groups were treated with $100 \mu \mathrm{M}$ ginsenoside Rg1, as described above. TUNEL assays (Roche, 1684795) were then performed according to the protocol provided by the manufacturer. Slides rinsed with PBS were counterstained with $1 \mathrm{mM}$ of Hoechst stain (Sigma, 861405). The fluorescein isothiocyanate (FITC)-labeled TUNEL-positive cells were imaged using the Synergy $^{\text {in }} 4$ Hybrid Microplate Reader system (BioTek) with $488 \mathrm{~nm}$ excitation and $530 \mathrm{~nm}$ emission wavelengths. The cells with green fluorescence were classified as apoptotic cells.

\section{Flow cytometric analysis}

Flow cytometry was carried out for nine model and nine treatment groups (allocated as in the TUNEL assay). H9c2 cells with or without Rg1 treatments were trypsinized and resuspended in cell culture medium; at least $10^{5}$ cells were collected. The cells were washed twice with PBS, then $5 \mu \mathrm{l}$ FITC-Annexin $\mathrm{V}$ and $5 \mu \mathrm{l}$ propidium iodide (PI) were added to the cells, and the cells were incubated in the dark at room temperature for $15 \mathrm{~min}$. Binding buffer was added, and cells were analyzed by flow cytometry within $30 \mathrm{~min}$.

\section{Immunoprecipitation assay}

A time-effect study was carried out for nine model and nine treatment groups (allocated as in the TUNEL assay). Whole-cell lysates were used for immunoprecipitation with antibodies against Beclin1. Ten micrograms of antibody was incubated with $1 \mathrm{ml}$ of cell lysate at $4{ }^{\circ} \mathrm{C}$ overnight. After the addition of Protein A/G-agarose beads, the incubation was continued for a further $4 \mathrm{~h}$. After centrifugation, the immunoprecipitates were extensively washed with lysis buffer 3-4 times and eluted with loading buffer. Bcl-2 antibodies were used in the subsequent Western blot experiments.

\section{Western blot analysis}

Western blot experiments were carried out for nine model and nine treatment groups (allocated as in the TUNEL assay). Protein samples were extracted from $\mathrm{H} 9 \mathrm{c} 2$ cells. Preparation of protein samples consisted of several steps, including splitting, centrifugation and boiling. Protein samples $(20 \mu \mathrm{g})$ were fractionated by sodium salt (SDS)Polyacrylamide gel electrophoresis (PAGE) (10 or $12 \%$ polyacrylamide gels), transferred to a polyvinylidene difluoride (PVDF) membrane and then blocked in $5 \%$ bovine serum albumin and prepared in a Tris-buffered saline (TBS) for $1 \mathrm{~h}$ at room temperature. The membranes were incubated with primary antibodies (GAPDH as a loading control) overnight at $4{ }^{\circ} \mathrm{C}$ then washed five times with $0.1 \%$ Tween-20 in Tris-buffered saline (TBS-T) for 30 min each. The membrane was then incubated with horseradish peroxidase (HRP)-conjugated secondary antibodies for $1 \mathrm{~h}$ at room temperature. After washing, the immunoreactive protein bands were developed by enhanced chemiluminescence (ECL), and the resulting membranes were imaged using the gel imaging system (BIO-RAD).

\section{FRET measurements}

FRET experiments were carried out for five groups, including one control group, one model group and three treatment groups $(25,50$ and $100 \mu \mathrm{M}$ ginsenoside $\mathrm{Rg} 1$ pretreatment, respectively). Experiments were performed in H9c2 cells stably co-expressing Cyan fluorescent protein (CFP) -BECN1 and Yellow fluorescent protein (YFP) -Bcl-2. The cells were washed with glucose-free Tyrode's solution three times and cultured in glucose-free Tyrode's solution with or without $\mathrm{Rg} 1$ treatment at $37^{\circ} \mathrm{C}$ until cell death. Fluorescence was measured with the microplate system every $10 \mathrm{~min}$ with the excitation wavelength of CFP and emission wavelength of YFP set at $436 \mathrm{~nm}$ and $527 \mathrm{~nm}$, respectively.

\section{Statistical analysis}

Quantitative data were expressed as mean \pm standard deviation (SD). Differences between experimental groups were examined by one-way analysis of variance (ANOVA), and means of two groups were compared using Student's $t$-test (paired, 2-tailed) by SPSS 18.0 software. For all analyses, $p<0.05$ was considered statistically significant.

\section{Results \\ Ginsenoside Rg1 inhibits starvation induced-apoptosis, contributing to cardioprotection}

During starvation, cell survival significantly decreased in a time-dependent manner between 30 and $240 \mathrm{~min}$ when compared with the 0 min (Additional file 1: Figure S1). At $240 \mathrm{~min}, 40 \%$ cell death had occurred, so this time point was deemed an adequate duration for serum and glucose deprivation in subsequent experiments. To evaluate whether Rg1 protects H9c2 cardiomyocytes under starvation conditions, cell viability was also measured after pretreatment with cultured media containing $\operatorname{Rg} 1$ at all three concentrations $(100,50,25 \mu \mathrm{M})$. Cell viability significantly increased at 120, 150, 180, 210 and 240 min with $\operatorname{Rg} 1$ 
treatment (Fig. 1a). Moreover, a dose-dependent effect was also observed between 120 and $240 \mathrm{~min}$ (Fig. 1a). Consequently, the higher, more effective dose of ginsenoside Rg1 $(100 \mu \mathrm{M})$ was chosen for all subsequent experiments.

Annexin V-FITC flow cytometric analysis showed that the population of Annexin $\mathrm{V}$ positive/PI negative cells increased in a time-dependent manner. Rg1 treatment decreased apoptosis by 5 to $15 \%$ between 60 and $240 \mathrm{~min}$ when compared to the corresponding time point of starvation model group $(P<0.05$ or $P<0.01$, Fig. $1 b)$. Similarly, TUNEL assay results showed that Rg1 treatment reduced the starvation-induced increase of TUNELpositive cells at all time points (Fig. 1c, d).

A time-dependent increase in active caspase 3 levels was observed between 0 and $120 \mathrm{~min}$ followed by a timedependent decline in both the model and Rg1 groups. In both cases, peak levels were observed at 120 min after induction of starvation. Rg1 treatment significantly decreased the levels of active caspase 3 by 39, 40, 37, 37, 44 and $59 \%$ at 90, 120, 150, 180, 210 and 240min after induction of starvation respectively when compared to the model group (Fig. 1e).

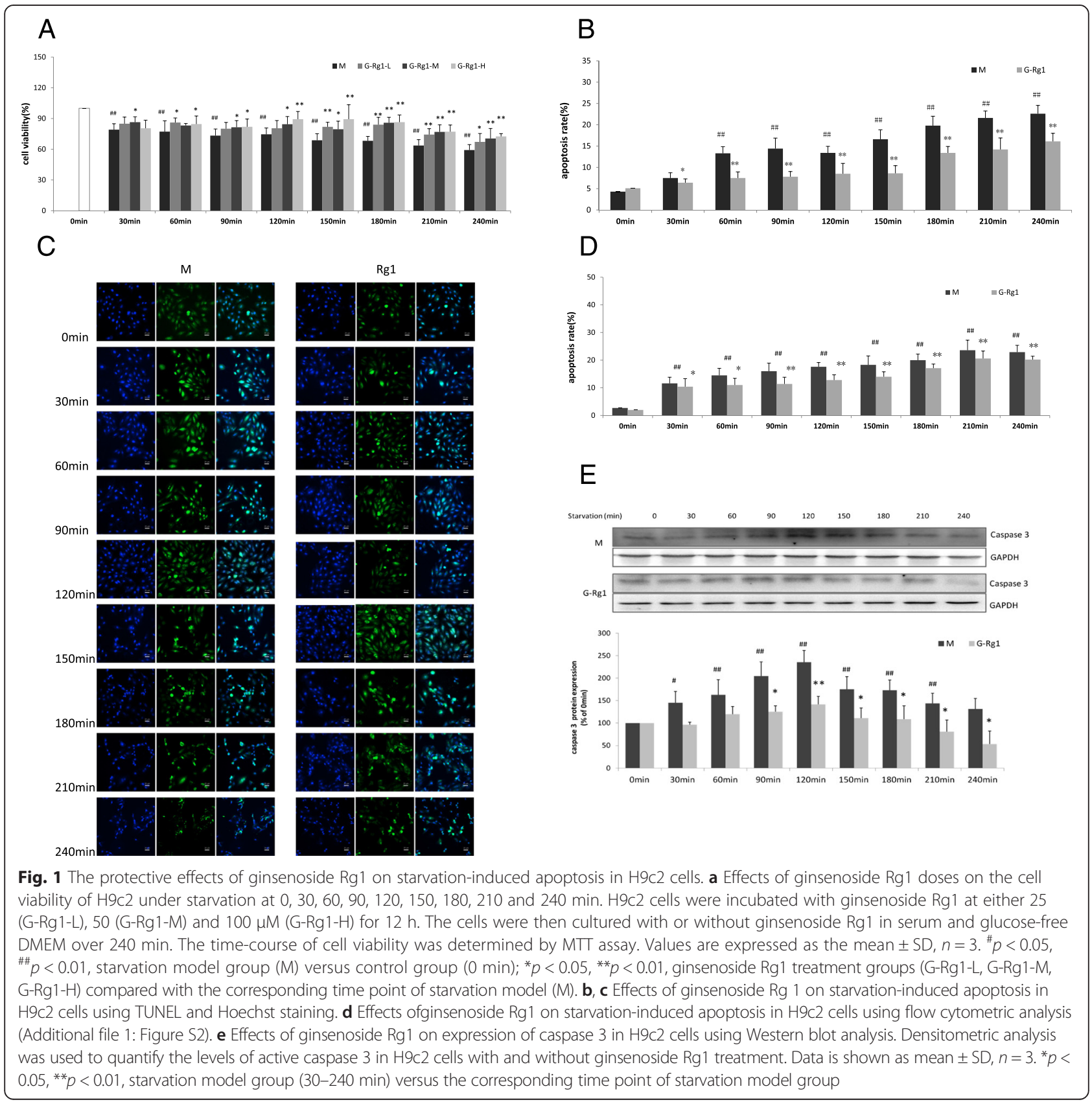


Rg1 promotes starvation-induced autophagy while inhibiting apoptosis in H9c2 cells

In the starvation model group, green fluorescence intensity persistently increased over $90 \mathrm{~min}$ accompanied by contraction of the cells. Four hours $(240 \mathrm{~min})$ after the induction of starvation, the cells contracted to a spherical shape, their movement stopped and the fluorescence was quenched. In the Rg1 treatment group, however, green fluorescence intensity began to increase at starvation $60 \mathrm{~min}$,then fluorescence was stable until quenched after starvation $840 \mathrm{~min}$ and the normal cell morphology was maintained over $420 \mathrm{~min}$ after starvation (Fig. 2a), suggesting that autophagic vacuolization may have occurred and cell death was delayed in response to ginsenoside Rg1 treatment.

As inefficient autophagic vesicle turnover (autophagosome-lysosome fusion and/or down-stream cargo degradation) can lead to autophagic vesicle accumulation [33], we proposed that $\operatorname{Rg} 1$ might induce autophagic vacuolization by impairing autophagic vesicle turnover. Therefore, protein levels of LC3 and p62 in H9C2 cells were assessed under starvation conditions at different time point following induction of starvation. Western blot analysis revealed that the LC3-II/LC3-I ratio was progressively up-regulated during starvation, achieving statistical significance at 90 min and peaking at $150 \mathrm{~min}$, before starting to decline (Fig. 2b), while p62 protein levels were progressively reduced under starvation (Fig. 2c). When compared with the corresponding time point of starvation model group, ginsenoside Rg1 treatment significantly increased the LC3-II/ LC3-I ratio by $42,37,25 \%$ at 30, 60 and 90 min respectively $(P<0.05$,or $P<0.01)$ and decreased p62 protein levels at 30,60, 90 and $120 \mathrm{~min}(P<0.05)$. These results suggest that $\operatorname{Rg} 1$ promotes autophagic activity in parallel with the inhibition of apoptosis in $\mathrm{H} 9 \mathrm{c} 2$ cells.

As illustrated in Fig. 2d, the expression of LC3-II was significantly elevated in the presence of both chloroquine(CQ) and Rg1 when compared to the model, CQ and

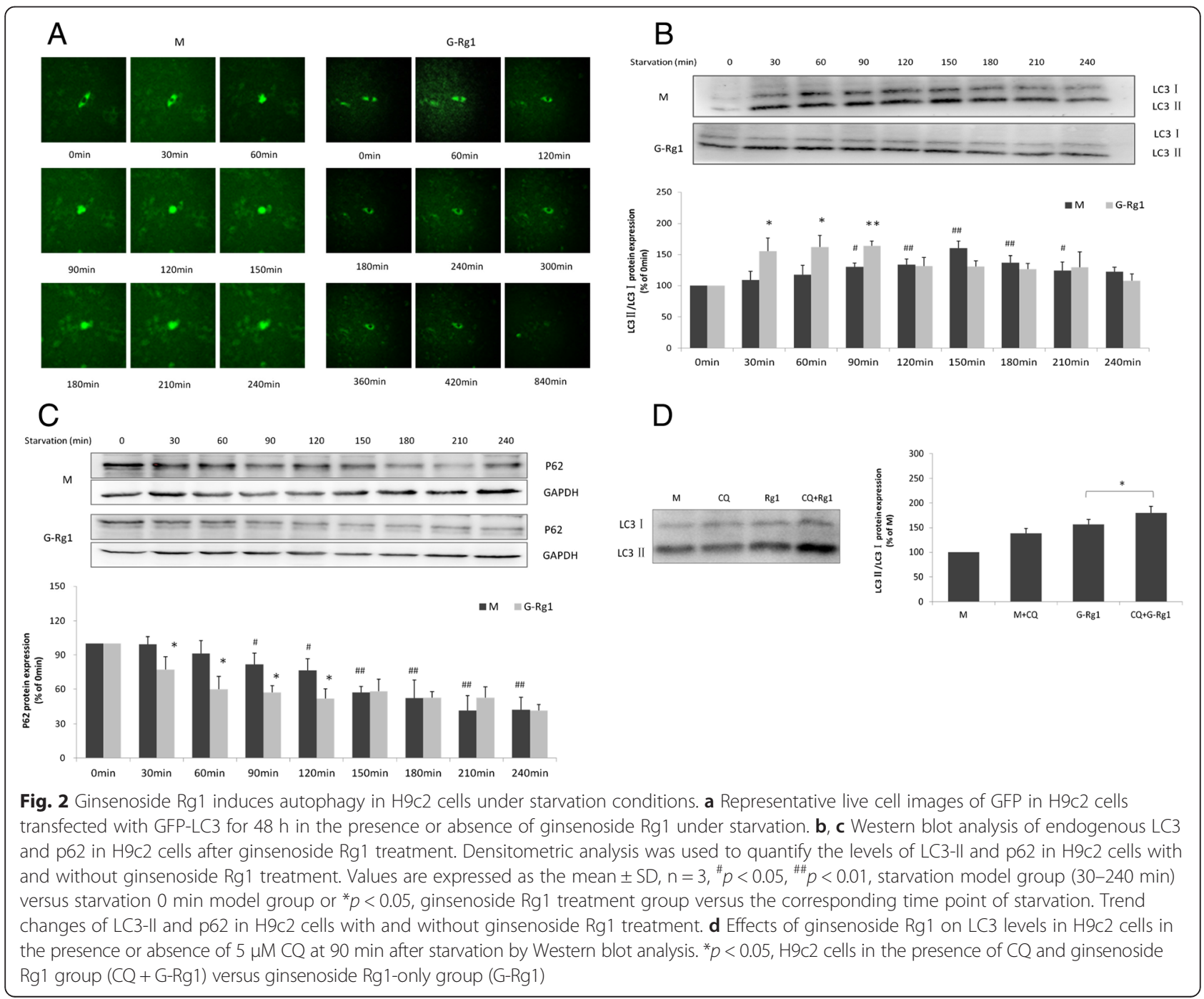


ginsenoside Rg1 treatment-only groups. This suggests a CQ-induced accumulation of autophagic vesicles induced by $\mathrm{Rg} 1$ treatment in $\mathrm{H} 9 \mathrm{c} 2$ cells under starvation. This result provides further evidence to support the concept that Rg1 promotes autophagy, without however significantly impairing autophagic vesicle turnover. (The effect of CQ on differet time pionts showed in Additional file 2: Figure S3).

\section{Rg1 mediates BECN1-Bcl- 2 interactions in H9c2}

Caspase 3 levels (Fig. 3a) increased in the presence of the inhibitor 3-MA and decreased in the presence of Rap at the same time point. 3-MA and Rap could inhibit or increase the expression of LC3-II of the model cell (Fig. 3b). The combined data suggested that promoting autophagy could suppress apoptosis and may be one of the mechanisms underlying the protective effect of $\operatorname{Rg} 1$ on starving H9c2 cells. This result also highlights a potential therapeutic target for reducing starvation-induced apoptosis via promoting autophagy.

Beclin1 expression was upregulated between 30 and 150 min after commencement of starvation and then began to decline to a statistically insignificant level (Fig. 4a). Meanwhile, no significant changes in Bcl-2 expression were noted in response to starvation. Treatment with Rg1 markedly increased levels of Beclin-1 and Bcl-2 at most time points. When compared to the corresponding time of starvation model group, $\operatorname{Rg} 1$ treatment significantly increased Beclin-1 protein levels by 24 to $88 \%$ between 90 and 240 min (Fig. 4a) and Bcl-2 protein levels by 87 to $147 \%$ between 90 and 210 min (Fig. 4b).

The immunoprecipitation and Western blot analyses showed that BECN1 levels immunoprecipitating with Bcl-2 steadily increased in a time-dependent manner during starvation while free $\mathrm{Bcl}-2$ levels decreased gradually. Conversely, Rg1 treatment upregulated $\mathrm{Bcl}-2$ while weakening the BECN1-Bcl-2 interaction in a timedependent manner (Fig. 5a). One hundred micrometer Rg1 is the optimal dosage for protecting $\mathrm{H} 9 \mathrm{C} 2$ cells from starvation. It upregulated the ratio of $\mathrm{Bcl}-2$ and BECN1-Bcl-2 by $35 \%$ (Fig. 5b).

In the starvation model group, YFP intensity started to increase at $240 \mathrm{~min}$ after starvation then peaked at 420 min and commenced a gradual decline, reaching a plateau at $480 \mathrm{~min}$ after starvation (Fig. 6a). Treatment with low does of $\operatorname{Rg} 1(25 \mu \mathrm{M})$, the YFP intensity markedly decreased from 280 to 540 min when compared to the corresponding time point of model group (Fig. 6b). Respectively, after middle $(50 \mu \mathrm{M})$ and high $(100 \mu \mathrm{M})$ does of Rg1 treatment, YFP intensity began to decrease at starvation 260 and 270 min (Fig. 6c, d). These results suggest that Rg1 produced similar dose-dependent changes in YFP intensity and the interaction between $\mathrm{BECN} 1$ and $\mathrm{Bcl}-2$ may have been weakened by ginsenoside $\operatorname{Rg} 1$.

\section{Discussion}

Previous studies have revealed multiple pharmacological effects of ginsenoside Rg1. The present study reports for the first time the dynamic regulatory effects of Rg1 on autophagy and the interactions between autophagy and apoptosis in H9c2 cells under starvation. These data provide evidence that $\operatorname{Rg} 1$ can partially inhibit the process of apoptosis by enhancing autophagy under continuous starvation conditions.

Autophagy is an evolutionarily conserved pathway that involves the sequestration and delivery of cytoplasmic materials to the lysosomes, where proteins are degraded and recycled [36]. It is involved in organelle turnover and bioenergetic management during starvation. In recent years,
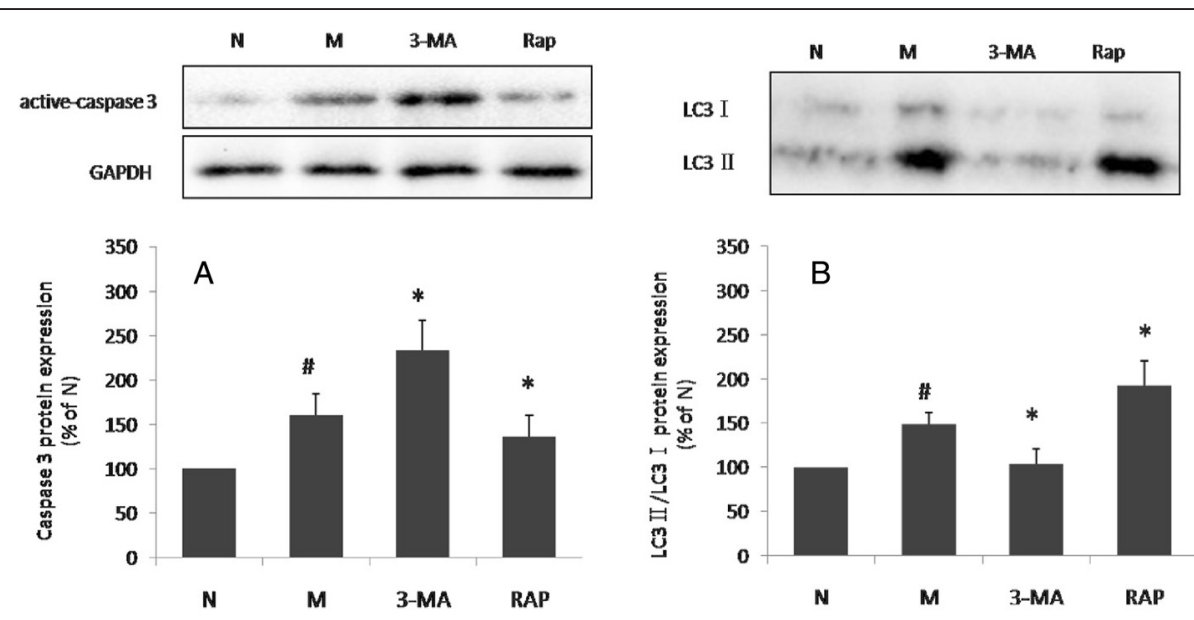

Fig. 3 Effects of 3-MA Rap on LC3 and active caspase 3 in H9c2 cells. H9c2 cells were treated with 3-MA for 24 h or Rap for 12 h under normal conditions and for 150 min under starvation. Expression of LC3 and active caspase 3 was determined by Western blot. Values are expressed as the mean $\pm \mathrm{SD}, n=3$. ${ }^{\#} p<0.05$ model group versus normal group; ${ }^{*} p<0.05$, ginsenoside $\mathrm{Rg} 1$ treatment group verse starvation model group (The effect of 3-MA and Rap on different time points showed in Additional file 2: Figures S4 and S5) 


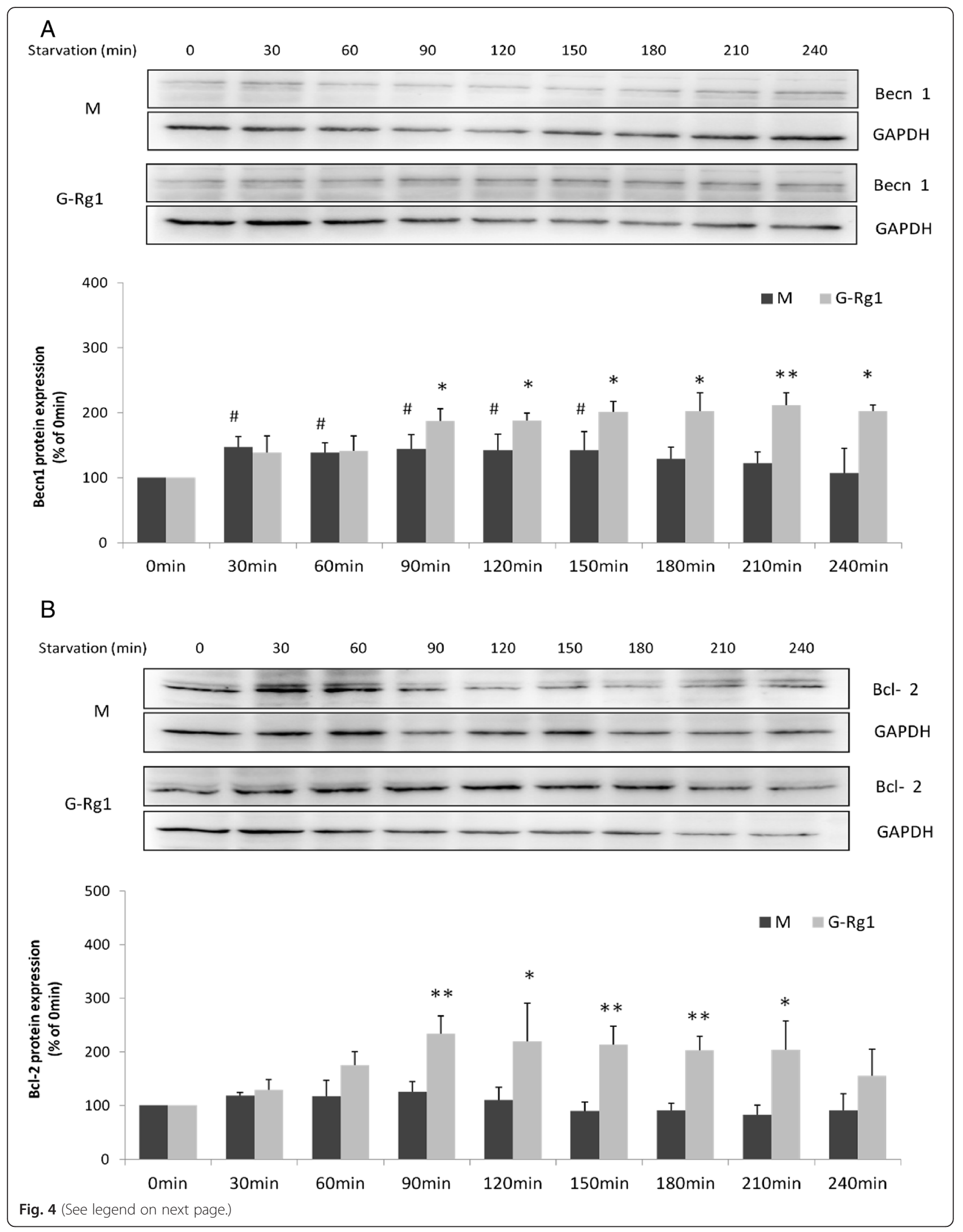


(See figure on previous page.)

Fig. 4 Increased expression of Beclin 1 and $\mathrm{BCl}-2$ in response to ginsenoside $\mathrm{Rg} 1$ in $\mathrm{H} 9 \mathrm{c} 2$ cells under starvation conditions. a, b Western blot analysis of endogenous Beclin 1 and $\mathrm{Bcl}-2$ in $\mathrm{H} 9 \mathrm{c} 2$ cells after treatment with and without ginsenoside $\mathrm{Rg} 1$. Values are expressed as the mean $\pm \mathrm{SD}, n=3$, $\# p<0.05,{ }^{\# \#} p<0.01$, starvation model group (M) verse control group (0 min) or ${ }^{*} p<0.05,{ }^{* *} p<0.01$, tarvation $30-240$ min model group verse starvation Omin model group or ${ }^{*} p<0.05,{ }^{* *} p<0.01$, ginsenos ide $\mathrm{Rg} 1$ treatment group (G-Rg1) verse the corresponding time point of starvation model group

the regulation and contribution of the autophagic process to cell metabolism has been characterized in great detail. As a process of consuming cellular components to generate energy, autophagy engages in a complex interconnection with apoptosis according to the nature of the stimulus and cell type. It either suppresses apoptosis by eliminating damaged organelles under cellular stress or sensitizes cells to apoptosis, acting as an energy source. There remains considerable debate as to the relationship between autophagy and apoptosis. The dynamic processes of autophagy and apoptosis have prompted the need to understand how autophagy and apoptosis interact at different stages and in response to various conditions.

In the starvation model in H9c2 cells described here, marker proteins of autophagy were initially evaluated. The conversion of LC3-I (18 kDa) to LC3-II $(16 \mathrm{kDa})$ is a strong biochemical marker for the induction of autophagy [37, 38]. The levels of LC3-II at different time points under starvation condition were thus measured. The LC3-II/LC3-I ratio was significantly increased at 90, $120,150,180$ and $210 \mathrm{~min}$ after starvation when compared to the control (Fig. 2b). This result is consistent with that of Han et al. [37] who reported that the accumulation of LC3-II in primary cardiomyocytes increased at 1, 2, 3 and $6 \mathrm{~h}$ under starvation conditions. The existence of an induced autophagic flux was also evaluated through the analysis of p62 levels, also called sequestosome 1 (SQSTM1). SQSTM1 is an ubiquitin-binding scaffold protein that is localized to the autophagosome via LC3-interaction and is specifically degraded by the autophagy-lysosome system [39]. p62 levels are thus inversely related to autophagic activity. The results of the present study showed that p62 levels were significantly decreased in response to starvation (Fig. 2c).

Apoptosis is the principal mechanism by which cells are physiologically eliminated in metazoan organisms [30]. In the present study, the protein expression levels of caspase 3, an indicator of apoptotic activity, were measured. These results revealed that apoptosis and autophagy occur simultaneously in $\mathrm{H} 9 \mathrm{c} 2$ cardiomyocytes under starvation conditions, but the dynamics of each process differ. For example, when the starvation-induced increases in LC3 expression reached their maximum, the caspase 3 levels began to decline (Fig. 1d, e). Significant changes in caspase 3 activity in cells incubated with 3MA or Rap were observed. In previous studies [12], 2mM 3-MA was shown to effectively block autophagic activity, while $50 \mathrm{nM}$ Rap effectively promoted autophagic activity in cardiomyocytes. The results of the present study showed that 3-MA enhanced starvation-induced caspase 3 activation, whereas Rap restrained its elevation, thus suggesting that autophagy promotion played a protective role in $\mathrm{H} 9 \mathrm{c} 2$ cardiomyocytes under starvation conditions (Fig. 3).

Rg1 has been demonstrated to have anti-ischemic, anti-hypoxic and pro-autophagic properties [13]. In this study, the effect of $\mathrm{Rg} 1$ on autophagy in $\mathrm{H} 9 \mathrm{c} 2$ cardiomyocytes was first investigated through determining the fluorescence intensity of the fusion protein GFP-LC3, which primarily represents the abundance of autophagosomes [40]. To date, few studies have reported the use of a real-time monitoring method for collection of the fluorescent signal. In fact, the real-time monitoring of green fluorescence in a single cell could provide vital information on the complete picture of GFP-LC3 changes in response to starvation over time. In the present study, Rg1 treatment not only increased fluorescence intensity but also delayed the time for fluorescence quenching by several hours. The existence of an induced autophagic flux by $\operatorname{Rg} 1$ was also evaluated through the analysis of LC3-II and p62 protein levels. Similarly, the results showed that Rg1 brought forward the high peak time of LC3-II/LC3-I and low peak time of p62, suggesting that Rg1 treatment promotes starvation-induced autophagic activities (Fig. 4). In addition, the effects of ginsenoside Rg1 on apoptosis were also evaluated over multiple time points. As measured by flow cytometry, Western blot and TUNEL assay, Rg1 effectively inhibited starvation-induced apoptosis in H9c2 cardiomyocytes at multiple time points.

To determine the mechanism underlying the regulatory effects of $\operatorname{Rg} 1$ on the relationship of autophagy and apoptosis in starving $\mathrm{H} 9 \mathrm{c} 2$ cells, the interaction of $\mathrm{Bcl} 2$ and BECN1 was investigated as an important link between apoptotic and autophagic activities. The BECN1 protein binds to $\mathrm{Bcl} 2$ through its $\mathrm{BH} 3$ domain. Under normal circumstances, the BH3-only domain of BECN1 may competitively disrupt the bringing of pro-apoptotic proteins to $\mathrm{Bcl}-2$ or $\mathrm{Bcl}-\mathrm{XL}$, thus preventing the induction of apoptosis [41]. Under stress conditions, Beclin 1, which is normally sequestered by Bcl-2, is released to induce autophagy. The results of the present study showed that $\operatorname{Rg} 1$ treatment upregulated the levels of Beclin 1 and Bcl-2 protein compared with the starvation model group at a slightly different time point. Immunoprecipitation is a 


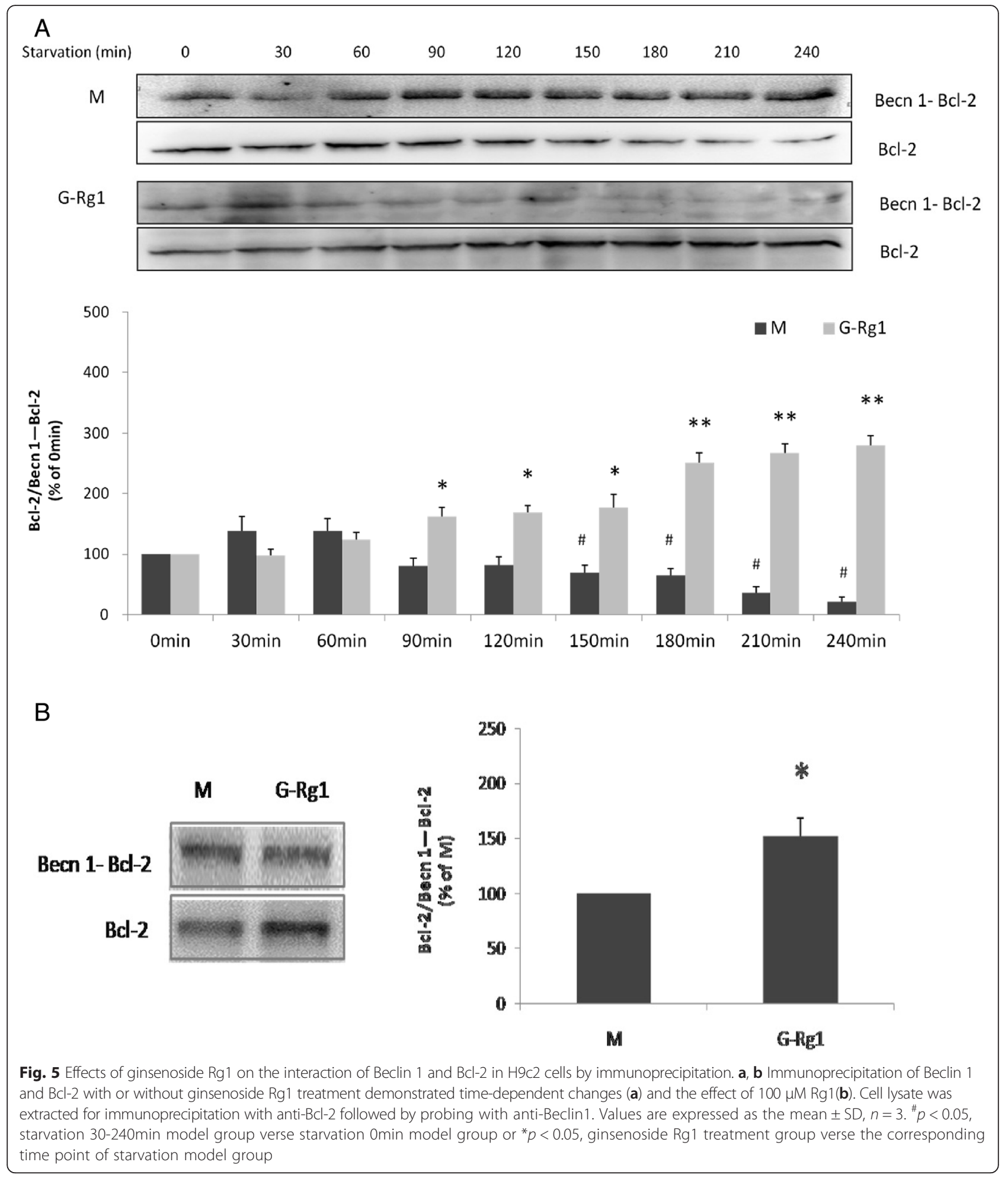

classic method for studies of protein interactions. In the present study, Rg1 promoted the dissociation of BECN1 and Bcl-2, increasing free BECN1 and Bcl-2 in the cytoplasm and consequently triggering the autophagic process in a dose- and time-dependent manner. FRET is a physical phenomenon by which energy from a donor fluorophore in an excited state is non-radiatively transferred to a neighboring acceptor fluorophore through dipole-dipole 


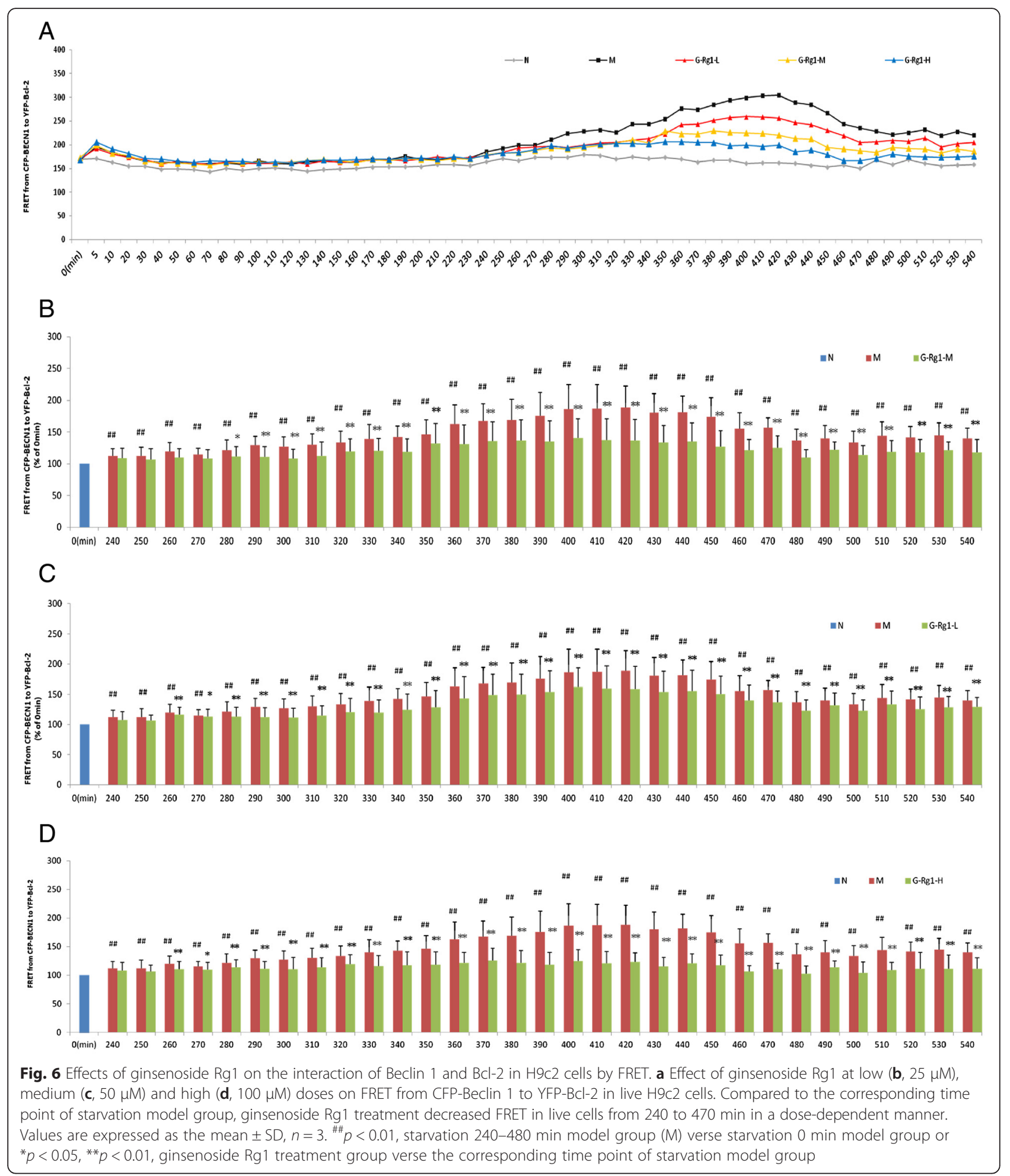

interactions. The strong dependence of FRET on distance enables the use of FRET to measure molecular interactions at a distance of 1-10 nm. The development of GFP and variants such as yellow fluorescent protein (YFP, 516 $\mathrm{Ex}_{\mathrm{Ex}} /$ $\left.527_{\mathrm{Em}}\right)$ and cyan fluorescent protein (CFP, 436 $\left.6_{\mathrm{Ex}} / 476_{\mathrm{Em}}\right)$ enables CFP/YFP to be used as a FRET pair. One of the most important findings of this study is that, by using the FRET assay, direct efficiency is provided that ginsenoside Rg1 treatment weakened the interaction between $\mathrm{Bcl}-2$ and Beclin1 in the starving H9c2 cardiomyocytes (Fig. 6). 


\section{Conclusions}

Our results support the theory that starvation contributes to the induction of autophagic activities in H9c2 cardiomyocytes. Ginsenoside Rg1 could prevent cellular apoptosis via initiating an autophagic survival response, during which time Rg1 could promote the expression of Beclin1 and Bcl-2 and weaken the interaction between Beclin1 and Bcl-2. This study has demonstrated for the first time a novel role for ginsenoside Rg1 in inducing a beneficial autophagic activity in cardiomyocytes under starvation.

\section{Additional files}

Additional file 1: Figure S1. Cell viability of H9c2 cells. Cell viability significantly decreased in a time-dependent manner between 30 and 240 min when compared with the control $(0 \mathrm{~min})$. Values are expressed as the mean $\pm S D, n=3 .{ }^{* *} p<0.01$, starvation model group verse control group. Figure S2. Effects of ginsenoside Rg1 on starvation-induced apoptosis in $\mathrm{H} 9 \mathrm{c} 2$ cells using flow cytometric analysis. (PDF $346 \mathrm{~kb}$ )

Additional file 2: Figure S3. The effect of CQ on different time points. The LC3 level significantly increased at 90 min $\left({ }^{*} p<0.01\right)$. Figure S4. The effect of 3-MA on different time points. The LC3 level significantly decreased at $12 \mathrm{~h}\left({ }^{*} p<0.05\right)$ and $24 \mathrm{~h}\left({ }^{*} p<0.05\right)$. Figure S5. The effect of Rap on different time points. The LC3 level significantly increased at 12 $\mathrm{h}\left({ }^{* *} p<0.01\right)$ and $24 \mathrm{~h}\left({ }^{*} p<0.05\right)$. (XLSX $\left.33 \mathrm{~kb}\right)$

\section{Abbreviations}

3-MA: 3-methyladenine; BECN1: Beclin1; CFP: cyan fluorescent protein; CQ: chloroquine; FITC: fluorescein isothiocyanate; FRET: fluorescence resonance energy transfer; GFP: green fluorescent protein; G-Rg1: ginsenoside Rg1; Rap: rapamycin; YFP: yellow fluorescent protein.

\section{Acknowledgments}

This research was supported by grants from the National Natural Science Foundation of China (Grant Nos. 81173582; 81473449; 81102679), the National Science \& Technology Major Project of China (Grant Nos.2012zx09301002-004-002; 2012zx09103201-049) and the National Basic Research Program of China (973 Program, 2015CB554400).

\section{Availability of data and materials}

The datasets supporting the conclusions of this article are included within the article and its additional file.

\section{Authors' contributions}

$\mathrm{DL}, \mathrm{JW}$ and $J \mathrm{~L}$ conceived, designed the experiments and drafted the paper $\mathrm{DL}, \mathrm{JH}$ and $\mathrm{JW}$ conducted the assays and analyses. All authors read and approved the final manuscript. JF and JL contributed to reagents/material/ analyses tools. BA and CD modified the language. All authors read and approved the final manuscript.

\section{Competing interests}

The authors declare that they have no competing interests.

\section{Ethics approval and consent to participate}

Not applicable.

\author{
Author details \\ ${ }^{1}$ Institute of Basic Medical Sciences, Xiyuan Hospital, China Academy of \\ Chinese Medical Sciences, Beijing Key Laboratory of Pharmacology of \\ Chinese Materia Medica, Hai Dian District, Beijing, China. ${ }^{2}$ Institute of Basic \\ Theory, China Academy of Chinese Medical Sciences, Dong Cheng District, \\ Beijing, China. ${ }^{3}$ National Institute of Complementary Medicine, University of \\ Western Sydney, Sydney, Australia.
}

Received: 22 July 2015 Accepted: 13 May 2016

Published online: 26 May 2016

\section{References}

1. Nemchenko A, Chiong M, Turer A, Lavandero S, Hill JA. Autophagy as a therapeutic target in cardiovascular disease. J Mol Cell Cardiol. 2011;51(4):584-93.

2. Terman A, Brunk UT. Autophagy in cardiac myocyte homeostasis, aging, and pathology. Cardiovasc Res. 2005;68(3):355-65.

3. Baehrecke EH. Autophagy: dual roles in life and death? Nat Rev Mol Cell Biol. 2005;6(6):505-10.

4. Hamacher-Brady A, Brady NR, Gottlieb RA. The interplay between pro-death and pro-survival signaling pathways in myocardial ischemia/reperfusion injury: apoptosis meets autophagy. Cardiovasc Drugs Ther/sponsored by the International Society of Cardiovascular Pharmacotherapy. 2006;20(6): 445-62.

5. Takagi H, Matsui $Y$, Sadoshima J. The role of autophagy in mediating cell survival and death during ischemia and reperfusion in the heart. Antioxid Redox Signal. 2007;9(9):1373-81.

6. Ge D, Jing Q, Meng N, Su L, Zhang Y, Zhang S, et al. Regulation of apoptosis and autophagy by sphingosylphosphorylcholine in vascular endothelial cells. J Cell Physiol. 2011;226(11):2827-33.

7. Han J, Pan XY, Xu Y, Xiao Y, An Y, Tie L, et al. Curcumin induces autophagy to protect vascular endothelial cell survival from oxidative stress damage. Autophagy. 2012;8(5):812-25.

8. Kang C, Avery L. To be or not to be, the level of autophagy is the question: dual roles of autophagy in the survival response to starvation. Autophagy. 2008;4(1):82-4.

9. Nguyen TM, Subramanian IV, Kelekar A, Ramakrishnan S. Kringle 5 of human plasminogen, an angiogenesis inhibitor, induces both autophagy and apoptotic death in endothelial cells. Blood. 2007;109(11):4793-802.

10. Xie Y, You SJ, Zhang YL, Han Q, Cao YJ, Xu XS, et al. Protective role of autophagy in AGE-induced early injury of human vascular endothelial cells. Mol Med Rep. 2011;4(3):459-64.

11. Yan L, Vatner DE, Kim SJ, Ge H, Masurekar M, Massover WH, et al. Autophagy in chronically ischemic myocardium. Proc Natl Acad Sci U S A. 2005;102(39):13807-12

12. Kobayashi S, Xu X, Chen K, Liang Q. Suppression of autophagy is protective in high glucose-induced cardiomyocyte injury. Autophagy. 2012;8(4):577-92.

13. Zhang ZL, Fan Y, Liu ML. Ginsenoside Rg1 inhibits autophagy in $\mathrm{H} 9 \mathrm{c} 2$ cardiomyocytes exposed to hypoxia/reoxygenation. Mol Cell Biochem. 2012; 365(1-2):243-50.

14. Kang C, You YJ, Avery L. Dual roles of autophagy in the survival of Caenorhabditis elegans during starvation. Genes Dev. 2007;21(17):2161-71.

15. Choi SH, Shin TJ, Lee BH, Chu DH, Choe H, Pyo MK, et al. Ginsenoside Rg3 activates human KCNQ1 K+ channel currents through interacting with the K318 and V319 residues: a role of KCNE1 subunit. Eur J Pharmacol. 2010; 637(1-3):138-47

16. Guo J, Gan XT, Haist JV, Rajapurohitam V, Zeidan A, Faruq NS, et al. Ginseng inhibits cardiomyocyte hypertrophy and heart failure via NHE-1 inhibition and attenuation of calcineurin activation. Circ Heart Fail. 2011;4(1):79-88.

17. Kim TH, Lee SM. The effects of ginseng total saponin, panaxadiol and panaxatriol on ischemia/reperfusion injury in isolated rat heart. Food Chem Toxicol. 2010;48(6):1516-20.

18. Lee JH, Choi SH, Lee BH, Shin TJ, Pyo MK, Hwang SH, et al. The effects of ginsenoside $\mathrm{Rg}(3)$ on human Kv1.4 channel currents without the N-terminal rapid inactivation domain. Biol Pharm Bull. 2009;32(4):614-8.

19. Yi XQ, Li T, Wang JR, Wong VK, Luo $P$, Wong IY, et al. Total ginsenosides increase coronary perfusion flow in isolated rat hearts through activation of PI3K/Akt-eNOS signaling. Phytomedicine. 2010;17(13):1006-15.

20. Zhao H, Lv D, Zhang W, Dong W, Feng J, Xiang Z, et al. Ginsenoside-Rb1 attenuates dilated cardiomyopathy in $\mathrm{CTnT(R141W)}$ transgenic mouse. J Pharmacol Sci. 2010;112(2):214-22.

21. Chen $H$, Yin J, Deng $Y$, Yang $M, X u L$, Teng $F$, et al. The protective effects of ginsenoside $\mathrm{Rg} 1$ against hypertension target-organ damage in spontaneously hypertensive rats. BMC Complement Altern Med. 2012;12:53.

22. Leung KW, Ng HM, Tang MK, Wong CC, Wong RN, Wong AS. GinsenosideRg1 mediates a hypoxia-independent upregulation of hypoxia-inducible factor-1alpha to promote angiogenesis. Angiogenesis. 2011;14(4):515-22.

23. Leung KW, Pon YL, Wong RN, Wong AS. Ginsenoside-Rg1 induces vascular endothelial growth factor expression through the glucocorticoid receptorrelated phosphatidylinositol 3-kinase/Akt and beta-catenin/T-cell factordependent pathway in human endothelial cells. J Biol Chem. 2006;281(47): 36280-8. 
24. Wang XD, Gu TX, Shi EY, Lu CM, Wang C. Effect and mechanism of panaxoside $\mathrm{Rg} 1$ on neovascularization in myocardial infarction rats. Chin J Integr Med. 2010;16(2):162-6.

25. Cheung LW, Leung KW, Wong CK, Wong RN, Wong AS. Ginsenoside-Rg1 induces angiogenesis via non-genomic crosstalk of glucocorticoid receptor and fibroblast growth factor receptor-1. Cardiovasc Res. 2011;89(2):419-25.

26. Akdemir F, Farkas R, Chen P, Juhasz G, Medved'ova L, Sass M, et al. Autophagy occurs upstream or parallel to the apoptosome during histolytic cell death. Development. 2006;133(8):1457-65.

27. Gajewska M, Gajkowska B, Motyl T. Apoptosis and autophagy induced by TGF-B1 in bovine mammary epithelial BME-UV1 cells. J Physiol Pharmacol. 2005;56(3):143-57.

28. Yin H, Liu Z, Li F, Ni M, Wang B, Qiao Y, et al. Ginsenoside-Rg1 enhances angiogenesis and ameliorates ventricular remodeling in a rat model of myocardial infarction. J Mol Med. 2011:89(4):363-75.

29. Boya P, Gonzalez-Polo RA, Casares N, Perfettini JL, Dessen P, Larochette N, et al. Inhibition of macroautophagy triggers apoptosis. Mol Cell Biol. 2005; 25(3):1025-40

30. Liang XH, Jackson S, Seaman M, Brown K, Kempkes B, Hibshoosh H, et al. Induction of autophagy and inhibition of tumorigenesis by beclin 1. Nature. 1999;402(6762):672-6.

31. Maiuri MC, Le Toumelin G, Criollo A, Rain JC, Gautier F, Juin P, et al. Functional and physical interaction between $\mathrm{BCl}-\mathrm{X}(\mathrm{L})$ and a $\mathrm{BH} 3$-like domain in Beclin-1. EMBO J. 2007;26(10):2527-39.

32. Oberstein A, Jeffrey PD, Shi Y. Crystal structure of the BCl-XL-Beclin 1 peptide complex: Beclin 1 is a novel BH3-only protein. J Biol Chem. 2007; 282(17):13123-32.

33. Kroemer G, Galluzzi L, Vandenabeele P, Abrams J, Alnemri ES, Baehrecke EH, et al. Classification of cell death: recommendations of the Nomenclature Committee on Cell Death 2009. Cell Death Differ. 2009;16(1):3-11.

34. Pattingre S, Tassa A, Qu X, Garuti R, Liang XH, Mizushima N, et al. Bcl-2 antiapoptotic proteins inhibit Beclin 1-dependent autophagy. Cell. 2005; 122(6):927-39.

35. Taylor RC, Cullen SP, Martin SJ. Apoptosis: controlled demolition at the cellular level. Nat Rev Mol Cell Biol. 2008;9(3):231-41.

36. Kabeya Y, Mizushima N, Ueno T, Yamamoto A, Kirisako T, Noda T, et al. LC3, a mammalian homologue of yeast Apg8p, is localized in autophagosome membranes after processing. EMBO J. 2000;19(21):5720-8.

37. Han X, Liu JX, Li XZ. Salvianolic acid B inhibits autophagy and protects starving cardiac myocytes. Acta Pharmacol Sin. 2011;32(1):38-44.

38. Komatsu M, Ichimura Y. Physiological significance of selective degradation of p62 by autophagy. FEBS Lett. 2010;584(7):1374-8.

39. Gozuacik D, Kimchi A. Autophagy as a cell death and tumor suppressor mechanism. Oncogene. 2004;23(16):2891-906.

40. Frommer WB, Davidson MW, Campbell RE. Genetically encoded biosensors based on engineered fluorescent proteins. Chem Soc Rev. 2009;38(10): 2833-41.

41. Lian J, Wu X, He F, Karnak D, Tang W, Meng Y, et al. A natural bhs minmeti induces autophagy in apoptosis-resistant prostate cancer viamo dulating bcl-2beclin1 interaction at endoplasmic reticlulm. Cell Differ. 2011;18(1):60-71.

\section{Submit your next manuscript to BioMed Central and we will help you at every step:}

- We accept pre-submission inquiries

- Our selector tool helps you to find the most relevant journal

- We provide round the clock customer support

- Convenient online submission

- Thorough peer review

- Inclusion in PubMed and all major indexing services

- Maximum visibility for your research

Submit your manuscript at www.biomedcentral.com/submit

Biomed Central 